\title{
ARBUSCULAR MYCORRHIZA IMPROVE APPLE ROOTSTOCK GROWTH IN SOIL CONDUCIVE TO SPECIFIC APPLE REPLANT DISEASE
}

\author{
H.J. RIDGWAY ${ }^{1}$, J. KANDULA ${ }^{2}$ and A. STEWART ${ }^{2}$ \\ ${ }^{\prime}$ Bio-Protection and Ecology Division, PO Box 84, Lincoln University, \\ New Zealand \\ ${ }^{2}$ National Centre for Advanced Bio-Protection Technologies, PO Box 84, \\ Lincoln University
}

Corresponding author: ridgwh@lincoln.ac.nz

\begin{abstract}
Specific apple replant disease (SARD) impairs the growth and establishment of trees in replanted apple orchards. Apple roots are normally colonised by arbuscular mycorrhizal fungi (AMF), which may have beneficial effects on plant growth. Four AMF inoculation treatments (three species of AMF, Glomus mosseae, Acaulospora laevis and Scutellospora calospora, and an uninoculated control) were applied to M26 apple rootstock seedlings in SARD and non-SARD soil. Of the fungi inoculated, S. calospora had the greatest beneficial effect in improving shoot and root dry weight and shoot length in SARD soil. More disease symptoms occurred on main and feeder roots in SARD soil and none of the inoculated AMF fungi reduced these. Both A. laevis and S. calospora significantly increased shoot length and gave a higher percentage of AMF-colonised roots in non-SARD soil. These results showed that AMF improve tolerance of apple to SARD and indicate that the beneficial effect is species specific. Characterisation of endogenous mycorrhizae in the soil identified S.pellucida. This is a new species record for New Zealand and provides the opportunity to determine whether the beneficial effect is specific to the genus Scutellospora. Keywords: apple, arbuscular mycorrhiza, specific apple replant disease (SARD), Glomus mosseae, Scutellospora calospora, Scutellospora pellucida, Acaulospora laevis.
\end{abstract}

\section{INTRODUCTION}

Specific apple replant disease (SARD) is a widespread disease that impairs the growth and establishment of plants in replanted apple orchards (Szabó et al. 1998). The disease is a major problem for growers trying to establish new orchards on sites where apples have been grown previously. The disease is characterised by stunted growth, yellowing of foliage, shortened internodes and small discoloured and rotting root systems. Growers incur significant costs including tree replacement costs and delayed and reduced yields. The cause of this disease has been suggested to include both abiotic factors and biotic factors, such as low nutrients, phytotoxins, Actinomycetes, fungal complexes and nematodes. However, fumigation of the soil has been shown to ameliorate the disease and indicates that the disease is primarily caused by biotic factors (Szabó et al. 1998).

As occurs in the majority of land plants, apples form associations with arbuscular mycorrhizal fungi (AMF). The role of this symbiosis in nutrient acquisition, particularly of phosphorus, is well known. However, AMF are also able to inhibit plant root diseases through mechanisms that are not yet well understood (Azcón-Aguilar \& Barea 1996). Among the mechanisms suggested are stimulation of host plant resistance pathways, interactions between AMF and pathogen mycelium and modification of the rhizosphere microflora. Prior research has shown that lower mycorrhizal colonisation of apple roots 
occurs in soils that are conducive to SARD (Caruso et al. 1989). The aim of this study was to determine the effect of AMF inoculation on SARD development in potted apple seedlings. As other studies have shown that a wide range of genera and species can be effective at inhibiting root pathogens, with no clear pattern, three different AMF genera were tested in this study.

\section{Soil and inoculum source}

\section{METHODS}

All soil used in the experiment was supplied by Dr Ian Horner, The Horticulture and Food Research Institute of New Zealand Limited, Hawke's Bay Research Centre. Soil conducive to SARD was sourced from an affected apple orchard and non-SARD soil, of a similar soil type, from a nearby pasture. Apple rootstock cuttings of variety M26 were obtained from Peakview Nursery Limited, Hastings, and rooted in sterile sawdust. The AMF spores were from sterile pot cultures produced using white clover 'Huia' as a bait plant. The three species, representing three different genera, were Glomus mosseae, Scutellospora calospora and Acaulospora laevis. These species had previously been identified using morphology and DNA sequence alignment of the ribosomal DNA region. The G. mosseae and A. laevis were both prepared at a concentration of 200 spores $/ 250 \mathrm{~g}$ of silica sand and the $S$. calospora at 150 spores/250 $\mathrm{g}$ of silica sand (due to limited availability of inoculum).

\section{Inoculation}

Each seedling was placed in a 2.5 litre plastic pot containing either SARD or non-SARD soil. The seedling was planted to a depth of approximately two-thirds of the way down the pot. The spores were applied in $250 \mathrm{~g}$ of sterile silica sand. The control treatment had silica sand without spores. To ensure that the inoculum was able to completely contact the new root system, a tube of approximately $5 \mathrm{~cm}$ diameter was placed around the roots and the sand/spore inoculum was placed in the tube. This tube was removed once the remaining space in the pot had been filled with the appropriate soil. Thus, the inoculum completely covered the root system and provided the mycorrhizae with the opportunity to colonise the roots prior to their exposure to the soil. A $2 \mathrm{~cm}$ thick layer of sterile potting mix was applied to the surface of the soil to prevent cross contamination between inoculated pots. The stem diameter of each seedling was recorded.

\section{Treatments}

There were eight treatments and ten replicate potted seedlings for each treatment. The trial was organised into five randomised blocks, each containing two pots of each treatment. The treatments were as follows: (1) non-SARD soil, (2) SARD soil, (3) SARD soil with G. mosseae, (4) non-SARD soil with G. mosseae, (5) SARD soil with S. calospora, (6) non-SARD soil with $S$. calospora, (7) SARD soil with A. laevis, (8) non-SARD soil with $A$. laevis. The pots were watered as required in a tray at the base of the pot. The plants were left to grow in the shadehouse for 8 months.

\section{Harvest}

After 8 months, the plants were destructively harvested. The plants were removed from the pots and washed gently to remove soil. The shoot length and shoot and root dry weights were recorded. The roots, main and feeder, were given a subjective disease score, where $0=$ no lesion (healthy) and $4=$ very dark brown to black (diseased). The total number of spores of AMF present in the soil was counted using the method of Brundrett et al. (1996). The total percentage root colonisation by AMF was assessed on feeder roots using the method of Brundrett et al. (1996). Results were analysed by ANOVA using GenStat Seventh Edition (Lawes Agricultural Trust, Rothamsted Experimental Station, UK, 2004).

\section{PCR and DNA sequencing}

Spore DNA was prepared by crushing eight sterile spores in $50 \mu 1$ of $5 \%$ Chelex resin (Chelex ${ }^{\circledR} 100$ Molecular Biology Grade Resin, 200-400 mesh Sodium Form, Bio-Rad Laboratories CA, USA), heating to $99^{\circ} \mathrm{C}$ for $20 \mathrm{~min}$, freezing at $-20^{\circ} \mathrm{C}$ and thawing. Each PCR contained $3 \mu \mathrm{l}$ of the Chelex extract, $10 \mathrm{mM}$ Tris $\mathrm{pH} 8.0,50 \mathrm{mM} \mathrm{KCl}, 200 \mu \mathrm{M}$ 
each of dGTP, dCTP, dATP and dTTP, $1.5 \mathrm{mM} \mathrm{MgCl}_{2}, 1.25 \mathrm{U}$ Taq DNA polymerase and five pmoles each of primers ITS1F ( ${ }^{5}$ CTTGGTCATTTAGAGGAAGTAA ${ }^{3}$ ) and ITS4 $\left({ }^{5}{ }^{\prime}\right.$ TCCTCCGCTTATTGATATGC ${ }^{3}$ ) in a $25 \mu$ l tube. Amplification of ribosomal DNA was done by heating to $94^{\circ} \mathrm{C}$ for $3 \mathrm{~min}$ and then 30 cycles of $94^{\circ} \mathrm{C}$ for $30 \mathrm{~s}, 50^{\circ} \mathrm{C}$ for $30 \mathrm{~s}$ and $72^{\circ} \mathrm{C}$ for $30 \mathrm{~s}$, followed by a final extension at $72^{\circ} \mathrm{C}$ for $7 \mathrm{~min}$. PCR products were sequenced in both directions at the Lincoln University Sequencing Facility.

\section{RESULTS}

Root and shoot weights were not significantly different between non-SARD and SARD soils but shoot length was significantly $(\mathrm{P}=0.008)$ lower in the SARD soil (Fig. 1). Root disease scores were significantly higher in the $\mathrm{SARD}$ soil $(\mathrm{P}<0.001)$ (Fig. 2). The effects of inoculation with AMF differed among the fungal species and between the soil types. Only S. calospora significantly increased shoot and root dry weight and shoot length in SARD soil (Fig. 1). Acaulospora laevis significantly increased root weight and shoot length in the non-SARD soil and $S$. calospora also increased shoot length in the non-SARD soil (Fig. 1). None of the inoculated fungi reduced disease scores in either SARD or non-SARD soil (Fig. 2). The percentage of apple roots colonised by AMF (both naturally-occurring and inoculated) was less in the SARD control compared to the non-SARD control (Fig. 1). Inoculation with S. calospora and with A. laevis significantly increased percentage of root colonisation in the non-SARD soil and inoculation with any of the three AMF resulted in increased root colonisation in the SARD soil to a level similar to the non-inoculated control in the non-SARD soil (Fig. 1). The total number of spores, irrespective of mycorrhizal species, in the soil at harvest was greater in nonSARD soil than in SARD soil (Fig. 2). In non-SARD soil, only A. laevis increased the number of spores, whereas, inoculation with S. calospora or G. mosseae decreased spore numbers. In SARD soil, the $S$. calospora treatment had spore numbers that were not significantly different from the non-SARD.

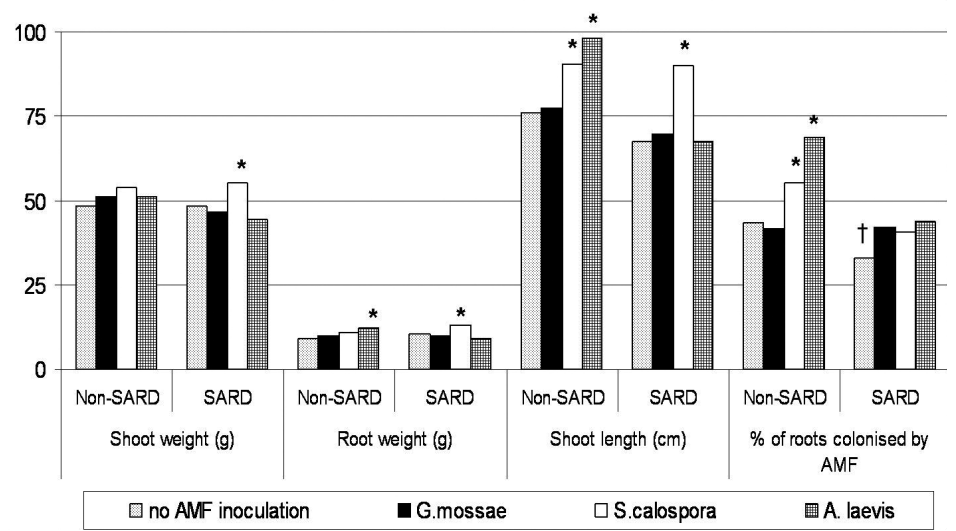

FIGURE 1: Plant growth parameter (shoot weight and root weight (g dry matter) and shoot length $(\mathrm{cm}))$ and percentage of roots colonised by arbuscular mycorrhizal fungi (AMF) for apple seedlings inoculated with three AMF and grown in two different soils. SARD soil was from an apple orchard where field symptoms of specific apple replant disease occurred and non-SARD soil was from a nearby pasture. For each variable, asterisks and crosses indicate treatments that were significantly greater or less than the non-inoculated, non-SARD control, respectively. 


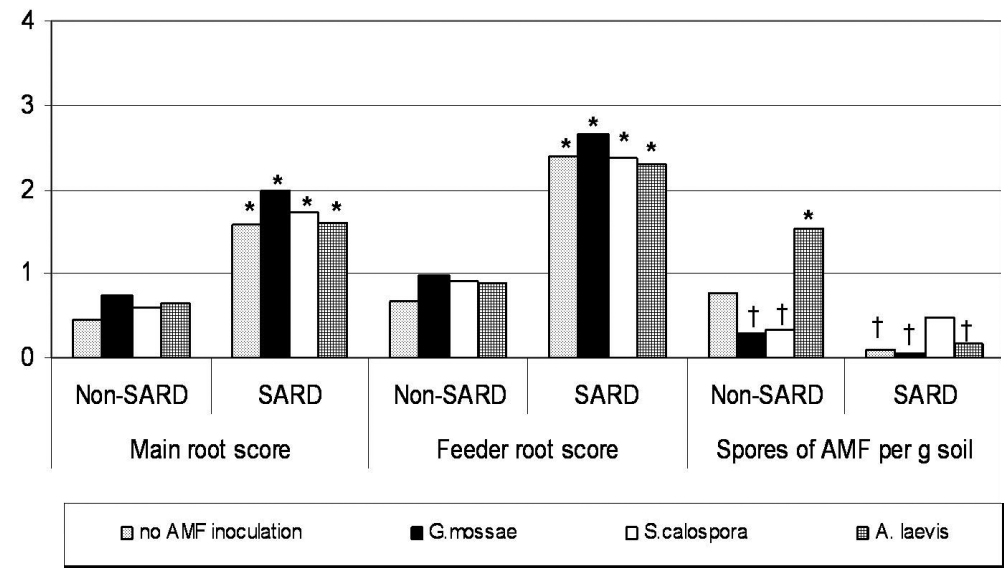

FIGURE 2: Apple seedling root disease scores, for main and feeder roots, and the number of AMF spores retrieved from two different soils planted with apple seedlings inoculated with three arbuscular mycorrhizal fungi (AMF).SARD soil was from an apple orchard where field symptoms of specific apple replant disease occurred and non-SARD soil was from a nearby pasture. For each variable, asterisks and crosses indicate treatments that were significantly greater or less than the non-inoculated, non-SARD control, respectively.

When the spores were sieved and counted to determine the final number of spores/g soil, spores of the genus Scutellospora were observed in all non-SARD treatments. Investigation of morphological features (Fig. 3) and sequencing of the ribosomal DNA showed that these spores had $95 \%$ sequence identity to S. pellucida. This is the first report of this species in New Zealand.

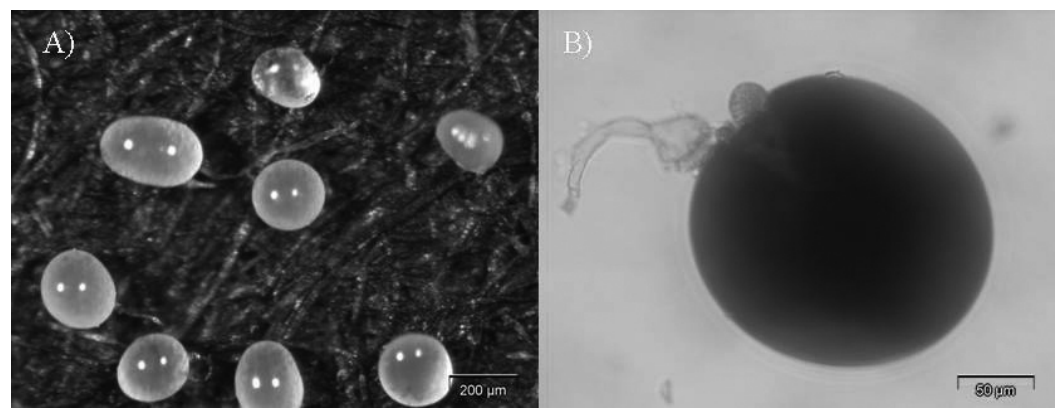

FIGURE 3: (a) Sterile $S$. pellucida spores on black filter paper. (b) $S$. pellucida spore stained in Melzer's reagent. 


\section{DISCUSSION}

This study demonstrated that of the three arbuscular mycorrhizal fungi used to inoculate apple seedlings, only S. calospora gave an improvement in plant growth in soil conducive to SARD. In non-SARD soil only A. laevis improved plant growth relative to the control. The relatively small effect of inoculation may have been due to the presence of other $\mathrm{AMF}$ and adequate levels of nutrients in the soil. Neither A. laevis nor G. mosseae had any effect on seedling growth in soil conducive to SARD. The ability of AMF to ameliorate plant root diseases has been reported previously (Azcón-Aguilar \& Barea 1996) and has been shown to vary between species and with soil type and environmental conditions. In SARD soil, the improved growth was not accompanied by an increase in the health of the seedling root system indicating that the mechanism is not simply via preventing the disease causing agent from infecting the roots. However, in SARD soil, inoculation with $S$. calospora stimulated root production in the apple seedlings and this was demonstrated by the increased root dry weight. The larger root system of the $S$. calospora inoculated apple seedlings is likely to have increased the total root mass available for water and nutrient uptake, decreasing the effect of the disease on seedling growth. The stimulation of root production suggests that as part of the symbiosis between $S$. calospora and apple, the fungus is able to affect plant growth and development and that this interaction is species specific.

In SARD soil there was no evidence of greater root colonisation by S. calospora relative to the other two AMF species or the control. This would suggest that differences in root and shoot dry weight are not due to differences in the biomass of AMF within the roots and indicates that the carbon requirement for the production of intraradical mycelium was likely to be similar between species. However, the measure of mycorrhizal colonisation was by incidence of fungal biomass counted using the gridline intersection method (Brundrett et al. 1996), which assumes that all AMF have similar colonisation patterns. Researchers have reported that the pattern of colonisation by Scutellospora spp. is very different from other genera (Dodd et al. 2000). Scutellospora spp. have localised and patchy colonisation unlike the extensive intraradical expansion of other species (Dodd et al. 2000). This may indicate that although the incidence of fungal biomass within the roots was similar, the amount of intraradical mycelium was actually less for S. calospora and was, therefore, less of a carbon drain on the plant.

It is also possible that extraradical mycelium development by $S$. calospora was less than that of the other two species, decreasing the carbon drain on the plant. Research has shown that root colonisation of clover by $S$. calospora peaks within the first 6 weeks and declines thereafter as spore production increases (Pearson \& Schweiger 1994). This pattern is different from other species such as Glomus spp., which maintain high levels of colonisation throughout plant growth. Thus, it is possible that significantly higher levels of colonisation by S. calospora were present earlier in the symbiosis and that this initially decreased the effect of the disease, allowing the plants to establish better and form a greater root mass. Sampling over the duration of the experiment rather than just at harvest would address this question.

During harvest and spore counting it was evident that spores of the genus Scutellospora were present in all treatments of the non-SARD soil. In previous analyses of agricultural soils, spores from the genus Scutellospora were rarely identified, unlike Glomus spp. and Acaulospora spp., despite their very obvious morphology. To confirm that their presence in all non-SARD treatments was not due to cross contamination with S. calospora the identity of the Scutellospora sp. was determined. Morphological and molecular analysis established that it was likely to be S. pellucida, which is a new record in New Zealand. Importantly, the addition of another Scutellospora to the culture collection will provide the opportunity to determine whether, in SARD infested soil, the enhanced root growth of the apple seedlings inoculated with $S$. calospora is genus or species specific. 


\section{ACKNOWLEDGEMENTS}

The authors would like to thank Ian Horner of The Horticulture and Food Research Institute of New Zealand Limited for supplying infested and non infested soils. This work was funded by the Foundation for Research, Science and Technology.

\section{REFERENCES}

Azcón-Aguilar C, Barea JM 1996. Arbuscular mycorrhizas and biological control of soil-borne plant pathogens - an overview of mechanisms involved. Mycorrhiza 6: 457-464.

Brundrett M, Bougher N, Dell B, Grove T, Malajczuk N 1996. Working with mycorrhizas in forestry and agriculture. Australian Centre for Agricultural Research, Canberra, Australia. $374 \mathrm{pp}$.

Caruso FL, Neubauer BF, Begin MD 1989. A histological study of apple roots affected by replant disease. Canadian Journal of Botany 67: 742-749.

Dodd JC, Boddington CL, Rodriguez A, Gonzalez-Chavez C, Mansur I 2000. Mycelium of arbuscular mycorrhizal fungi (AMF) from different genera: form, function and detection. Plant and Soil 226: 131-151.

Szabó K, Winkler H, Petzold H, Marwitz R 1998. Evidence for the pathogenicity of actinomycetes in rootlets of apple seedlings from soils conducive to specific apple replant disease. Acta Horticulturae 477: 55-58.

Pearson JN, Schweiger P 1994. Scutellospora calospora (Nicol. \& Gerd.) Walker \& Sanders associated with subterranean clover produces non-infective hyphae during sporulation. New Phytologist 127: 697-701. 